\title{
Evaluación de Percepciones Sobre la Interdisciplinariedad: Validación de Instrumento para Estudiantes de Educación Superior
}

\author{
Itzel Hernandez-Armenta( ${ }^{(1)}$ y Angeles Dominguez ${ }^{(1,2)}$ \\ (1) Tecnológico de Monterrey, México. (e-mail: armenta.itz@gmail.com) \\ (2) Universidad Andrés Bello, Santiago, Chile. (e-mail: angeles.dominguez@itesm.mx)
}

Recibido Jun. 21, 2018; Aceptado Ago. 21, 2018; Versión final Oct. 30, 2018, Publicado Jun. 2019

\begin{abstract}
Resumen
Este estudio presenta la construcción y validación (análisis de confiabilidad y validez) de un instrumento de medición de la percepción sobre la interdisciplinariedad para estudiantes universitarios. Se contó con la participación de 279 estudiantes de diversos programas académicos y cursando alguna materia de segundo año de educación superior. El instrumento consiste en trece reactivos evaluados en escala Likert (coeficiente de alfa de Cronbach $=0.704$ ), clasificados en tres dimensiones relativas a la percepción de competencias para la interdisciplinariedad (mostrando correlación de Pearson moderada para cada dimensión). Este trabajo aporta un test validado para medir la percepción que tienen los estudiantes de cualquier campo disciplinar sobre la integración del conocimiento en su experiencia educativa. El uso de este instrumento provee argumentos sobre la relevancia del enfoque interdisciplinario en la formación universitaria.
\end{abstract}

Palabras clave: interdisciplinariedad; percepción; validación; instrumento de medición; educación superior

\section{Assessment of Students' Perceptions About Interdisciplinarity: Instrument Validation for Higher Education Students}

\begin{abstract}
The present work shows the construction and validation (reliability and validity analysis) of a measurement instrument for the perception of interdisciplinarity in higher education students. A total of 279 students enrolled in a second-year course from various academic programs participated in the study. The instrument consists of a survey of thirteen items graded in a Likert scale (Cronbach's alpha $=0.704)$, classified in three dimensions related to the perception of competences for interdisciplinarity (showing a moderated Pearson correlation for every dimension). This study contributes with a validated test to measure perceptions that students from any disciplinary field have about knowledge integration in their educative experiences. The use of this instrument supports the relevance of an interdisciplinary approach in university formation.
\end{abstract}




\section{INTRODUCCIÓN}

Los fenómenos de integración del conocimiento están presentes en una gran variedad de contextos; han sido estudiados desde problemáticas que competen a la construcción del conocimiento científico y que responden a las necesidades de cooperación entre actores de la ciencia (Visholm et al., 2012), así como en entornos educativos de distintos niveles académicos y en los que se presentan una serie de retos para la educación del presente. Los enfoques educativos interdisciplinarios buscan que el conocimiento se integre y trascienda sus fronteras para facilitar la experiencia de aprendizaje, minimizando la fragmentación de unidades disciplinares e innovando así la educación (Broggy et al., 2017). La interdisciplinariedad, por lo tanto, es relevante para la educación superior a medida que el mundo cobra consciencia de su interdependencia y se conecta, permitiendo la experiencia del conocimiento desde distintos puntos de vista (Davies y Devlin, 2010; Tarrant y Thiele, 2016). Esta experiencia involucra el florecimiento de competencias para la interdisciplinariedad, como el desarrollo de conocimientos, habilidades y actitudes para el intercambio y ruptura de los conocimientos parcelados disciplinares en búsqueda de la integración (Parker, 2010). Piaget (1972) empleó el término interdisciplinariedad para explicar las relaciones recíprocas y de intercambio entre distintas áreas del conocimiento. Por su parte, Morin (1999) propone que la interdisciplinariedad busca una integración natural del saber: si se piensa en él como un gran "tejido", los componentes estructurales serían fibras entrelazadas, cada una engendrada en el marco de una disciplina; fibras que respetan su individualidad y valor propio sin descuidar su papel dentro de la totalidad. La idea de tejidos de conocimiento estimula un entendimiento holístico y superior de los saberes que no sólo evoca y muestra estructuras similares entre ellos, sino que de acuerdo a Morin (2008) dispone a la mente a esclarecer un "todo" que no se reduce a la suma de sus partes constitutivas.

No obstante, las integraciones no se limitan al plano llano de las fibras del conocimiento cuando se sitúan en contextos educativos; son los actores involucrados quienes experimentan los fenómenos de integración y sus consecuencias. Estos actores educativos han sido ampliamente analizados en entornos pedagógicos de primera infancia y de educación básica, entendiendo la naturalidad de la integración de conocimientos como indispensable para el desarrollo inicial del pensamiento (Deneme y Ada, 2012; Lenoir y Hasni, 2016; Wood, 2010). De la profunda investigación en esos niveles educativos se vincula inherentemente la construcción de puentes cognitivos al papel fundamental de las interacciones sociales escolares (Vygotsky, 1962); toda internalización de significados asociados a la construcción del conocimiento es intrínseca a las prácticas humanas, el aprendizaje es construido y reconstruido a partir de interacciones sociales y con el entorno, y es transmitido de la misma forma (Vygotsky, 1978). La educación interdisciplinaria, según Morin (1999), cultiva aptitudes naturales que ayudan contextualizar los saberes para lograr integrarlos en sus conjuntos naturales, es decir que prepara no sólo para entender el conocimiento sino para construirlo colectivamente en la complejidad de la que surge y a la que complementa, para comprendernos a nosotros mismos y a los demás, y para afrontar la incertidumbre que conlleva ser humano; por ello, la educación interdisciplinaria se suscribe tanto dentro de un paradigma epistemológico constructivista social, como a las corrientes pedagógicas correspondientes al mismo (Ledoux y McHenry, 2004).

La consolidación de los tejidos de conocimiento se da a lo largo de la vida y, en particular, en la formación universitaria. La complejidad de los problemas de la actualidad requiere de soluciones innovadoras y efectivas, por lo que algunas universidades incorporan programas para promover en los estudiantes habilidades de síntesis e integración de información de diferentes disciplinas (Knight et al. 2012). Asimismo, se ha estudiado que el establecimiento de redes de colaboración y diálogo entre expertos en distintos campos del conocimiento promueve espacios de aprendizaje colectivo (Feng, 2012). Además, desarrolla habilidades de innovación y reflexión del impacto social encontradas de la comparación entre estudiantes de educación superior (Contreras-Velásquez et al., 2017), dándole a la educación interdisciplinaria bases que fundamentan su implementación y estudio. La educación interdisciplinaria abre paso a otras maneras de abordar problemas reales y replantear soluciones. Debido a que la interdisciplinariedad integra información, metodologías y técnicas, conjuntos de habilidades o perspectivas teóricas de dos o más disciplinas, es posible aplicar las integraciones para entender y abordar problemas complejos reales, así como para relacionar teorías abstractas con situaciones y casos específicos (Tarrant y Thiele, 2017). Existe evidencia sobre el impacto que tiene la educación interdisciplinaria en los estudiantes, tal es el caso de Costa et al. (2015), quiénes reportaron que, al abordar problemas reales de la ciencia, tecnología y ecología, los estudiantes desarrollaban gusto por la relación de estos asuntos con su experiencia diaria, así como con los temas de actualidad y de gran impacto en la sociedad.

Lattuca et al. (2004) identifican antecedentes del reto para evaluar las diferencias de aprendizaje entre cursos que integran el conocimiento y aquellos que mantienen enfoques disciplinares. Este problema sigue vigente pues a la luz de las distintas declaraciones de los beneficios educativos del estudio interdisciplinar, es primordial entender si los estudiantes de carreras interdisciplinarias experimentan ganancias similares en el aprendizaje comparados con estudiantes en programas basados en una disciplina (Lattuca et al., 2017). 
En respuesta a su propio llamado a la comunidad académica, Lattuca et al. (2013) elaboraron un instrumento para evaluar las competencias interdisciplinarias en estudiantes de ingeniería. El instrumento identifica tres factores: 1) habilidades interdisciplinarias, refiriéndose a la evaluación de la apreciación de las distintas perspectivas disciplinares y no disciplinarias, el reconocimiento de las limitaciones de cada disciplina, la evaluación de la efectividad de aplicación interdisciplinaria en los cursos universitarios, la habilidad para encontrar terrenos comunes entre disciplinas y la habilidad para integrar holísticamente aquellos conocimientos particulares de cada disciplina para explicar mejor los fenómenos de una manera superior a una suma de conceptos, 2) comportamiento reflexivo, el cual evalúa la actitud reflexiva para identificar la complejidad de problemas del conocimiento y 3) reconocimiento de perspectivas disciplinarias, que mide la competencia de reconocer la existencia de límites disciplinares, así como de encontrar a partir de obstáculos epistemológicos relaciones entre disciplinas y los campos en los que estas pueden compartir un trabajo colaborativo.

Pensando en la clasificación propuesta por Lattuca et al. (2013) y en la necesidad de evaluar las competencias interdisciplinarias, surge interés por dirigir la evaluación hacia la percepción de la interdisciplinariedad en una de sus dimensiones, conocimientos, habilidades y actitudes, desde la perspectiva del estudiante. A partir de esta categorización, se construye el Test de percepción sobre la interdisciplinariedad, el cual tiene la intensión de aportar una herramienta de información y de análisis sobre la percepción que tienen los estudiantes sobre las competencias relativas a la integración del conocimiento en su vida académica. Para Fernandes (2012), la valoración de un objeto o situación puede reflejar la apreciación, interés, convicción o preferencia de este en comparación con otro, pues es una estructura conceptual personal que se marca una pauta para la acción y dirección de la conducta. De ahí la importancia de proporcionar un instrumento para identificar esas estructuras conceptuales de la percepción que los estudiantes tienen sobre la interdisciplinariedad y sus competencias para experimentarla.

\section{MÉTODO}

El presente trabajo de investigación expone la construcción y validación de un instrumento cuantitativo para la medición de la percepción sobre la interdisciplinariedad dirigido a estudiantes universitarios, sin restricciones relativas a la carrera o área disciplinar en la que se estén especializando. Esto permite, además de su aplicación a gran escala dentro de instituciones educativas, su uso en estudiantes de distintos enfoques disciplinares pues se pretende que este instrumento aporte una manera de conocer la percepción sobre el aprendizaje integrador en contextos universitarios desde el punto de vista del que aprende. Para la construcción del instrumento, se siguió un proceso exhaustivo de selección, traducción y readaptación contextual de reactivos pertenecientes a instrumentos ya validados en la literatura, como se muestra en la figura 1.

De la recuperación de documentos de investigación sobre la evaluación de la interdisciplinariedad, se consideraron diez investigaciones realizadas entre el 2011 y 2017 que tuvieran el objetivo de medir diversos factores de la interdisciplinariedad en diferentes contextos educativos. Posteriormente se identificaron y clasificaron las dimensiones propuestas por dichas aportaciones de acuerdo a la naturaleza de su objeto de estudio, englobándolas en tres dimensiones sugeridas en concordancia a la evaluación hecha sobre el desarrollo de competencias: a) evaluación del conocimiento: Reconocimiento de perspectivas disciplinares (Lattuca et al., 2013), experiencia holística de aprendizaje y aprendizaje interdisciplinario (Self y Baek, 2017), experiencia interdisciplinaria en el laboratorio (Walczak y Van Wylen, 2015), requerimiento curricular y estructura organizacional para la interdisciplinariedad (Knight et al., 2012) y la escala de interdisciplinariedad (Cezarino et al., 2015); b) evaluación de habilidades: habilidades interdisciplinarias (Lattuca et al., 2013), pensamiento interdisciplinario (Liu et al., 2011), pensamiento crítico y necesidad de cognición (Lattuca et al., 2017); y c) evaluación de actitudes: comportamiento reflexivo (Lattuca et al., 2013), actitudes positivas hacia la alfabetización (Lattuca et al., 2017), pensamiento reflexivo (Tutticci et al., 2016), capacidad interdisciplinaria, relaciones interpersonales, autonomía y compromiso académico (Koch et al., 2017), y colaboración interdisciplinaria (Miles y Rainbird, 2015).

Posteriormente se filtraron (A, figura 1) sólo aquellos trabajos que compartieran el ser dirigidas a la evaluación a nivel universitario, documentar esta evaluación en diferentes contextos disciplinares, tener un enfoque cuantitativo y ser autogestionadas por los estudiantes. Además, se tomaron en cuenta únicamente aquellas publicaciones que sí compartían la información completa de la composición de sus instrumentos de medición, ya que, la mayoría de las investigaciones sobre evaluación de la interdisciplinariedad se han elaborado en inglés y en el proceso de crear o adaptar un instrumento de naturaleza cuantitativa es de suma importancia cuidar factores que puedan entorpecer la validez de este, por lo que el hecho de hacer una traducción implica ya la necesidad de revalidación. 
De este primer filtro, se tomaron en cuenta 64 reactivos pertenecientes a cinco aportaciones de la literatura, clasificados por sus respectivos autores en las siguientes dimensiones: medición de competencias de interdisciplinariedad (Lattuca et al., 2013), experiencia (interdisciplinaria) en espacios de laboratorio (Walczak y Van Wylen, 2015), evaluación de proyectos interdisciplinarios (Koch et al., 2017), experiencia de aprendizaje holístico (Self y Baek, 2017) y pensamiento reflexivo (Tutticci et al., 2017).

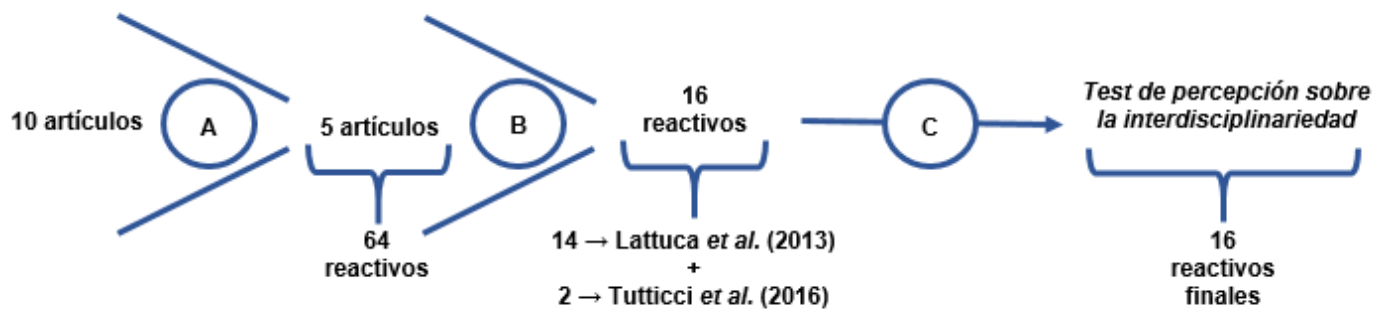

Fig. 1: Ruta metodológica para la construcción del Test de percepción sobre la interdisciplinariedad para su posterior validación.

Del listado de reactivos originales de las fuentes antes mencionadas se llevó a cabo un ejercicio de lectura en el idioma original para evaluar la posibilidad de sustitución de especificidad contextual e intensión de cada enunciado, considerando que se elaboraría un instrumento aplicable a cualquier estudiante universitario independientemente del área disciplinar en la que se desempeñara. De esta lectura se determinó que los reactivos de Walzack y Van Wylen (2015), Koch et al. (2017) y Self y Baek (2017) coincidían con las ideas abordadas en el instrumento propuesto por Lattuca et al. (2013), encontrando paralelismo entre los enunciados y decidiendo entonces preservar este último en su totalidad, partiendo de catorce enunciados. Se observó que, aunque se trataba de un instrumento de auto-reporte, su investigación reportaba evaluar las competencias de cada una de las dimensiones que componen al pensamiento interdisciplinario y que lo que reflejaban sus reactivos era la apreciación de la interdisciplinariedad a través de la opinión del estudiante, adaptándose mejor a un instrumento enfocado a la medición de la percepción. Además, a partir de esta segunda depuración ( $B$, figura 1) se encontró un desbalance en la cantidad de reactivos por dimensión del instrumento de Lattuca et al. (2013) a pesar de reportar una confiabilidad y precisión adecuadas para los fines de este trabajo. Por ello, se decidió complementar la dimensión menos proporcionada, relativa a las actitudes sobre la interdisciplinariedad, con dos reactivos pertenecientes al trabajo de Tutticci et al. (2017) con enunciados sobre el pensamiento reflexivo.

Finalmente, se hizo una traducción y adaptación del uso del lenguaje y vocabulario (C, figura 1) de cada uno de sus reactivos considerando que se elaboraría un instrumento dirigido a estudiantes universitarios indistintamente a su formación disciplinar, enriqueciendo la traducción de cada una de las oraciones. Por otra parte, se reclasificaron y reagruparon sus reactivos rigurosamente dentro de cada una de las dimensiones que se deseaban medir relativas a la percepción de competencias para la interdisciplinariedad (conocimientos, habilidades y actitudes), construyendo un nuevo instrumento de medición de acuerdo con esa categorización. Se consideró para esta fase de la elaboración del instrumento que algunos de los reactivos estuvieran redactados de manera invertida para contribuir a criterios de confiabilidad, ya que otra de las observaciones hechas sobre el trabajo de Lattuca et al. (2013) indicaba que todos los reactivos propuestos estaban redactados como enunciados positivos. Adicionalmente, se realizó una refinación cualitativa con nueve estudiantes de características demográficas similares a los participantes para la validación del instrumento, con la finalidad de identificar incongruencias de vocabulario y de comprensión de los reactivos ( $\mathrm{C}$, figura 1). Para la adaptación de las preguntas se tomó en cuenta, además de la traducción al español, la sintaxis y claridad con la que era expresada la idea de cada enunciado. Se evitó el uso de vocabulario especializado, así como enunciados tendenciosos que dieran mensajes implícitos y que evitaran conocer fielmente la opinión del encuestado.

El instrumento propuesto en este trabajo se compone de 16 ítems (tabla 1): cuatro que definen la dimensión de percepción de conocimientos disciplinares, ocho que definen la dimensión de percepción de habilidades para la interdisciplinariedad y cuatro que definen la dimensión de actitudes reflexivas (tabla 2). Las opciones de respuesta corresponden a una escala de Likert de cinco puntos: 1) completamente en desacuerdo, 2) en desacuerdo, 3) ni de acuerdo ni en desacuerdo, 4) de acuerdo, 5) completamente de acuerdo. El orden de los reactivos se decidió de manera que estuvieran desordenados respecto a su clasificación en cada una de las dimensiones evaluadas, pero manteniendo un "orden de lectura natural". Es decir, de R1 a R8 hacen referencia a la solución de problemas, de R9 a R12 a la identificación de vínculos entre disciplinas académicas y de R13 a R16 sobre hábitos de pensamiento. Aunque las dimensiones analizadas en este instrumento no están categorizadas de esa manera, otorgan al estudiante un camino a seguir a través del test. 
Tabla 1: Reactivos del Test de percepción sobre la interdisciplinariedad.

\begin{tabular}{|c|c|}
\hline Reactivo & Enunciado \\
\hline $\mathrm{R} 1$ & $\begin{array}{l}\text { Disfruto pensar cómo diferentes áreas de estudio abordan los mismos problemas siguiendo caminos } \\
\text { distintos. }\end{array}$ \\
\hline $\mathrm{R} 2$ & $\begin{array}{l}\text { Cuando un profesor experto en su materia da una explicación a una problemática, soy bueno(a) } \\
\text { identificando si el problema podría resolverse con enfoques de otras disciplinas académicas. }\end{array}$ \\
\hline R3 & $\begin{array}{l}\text { Al resolver problemas específicos de mi carrera, recurro frecuentemente a profesores y expertos en otras } \\
\text { áreas. }\end{array}$ \\
\hline R4 & Frecuentemente me detengo a pensar si voy "bien" o "mal" en el proceso de resolver un problema. \\
\hline R5 & $\begin{array}{l}\text { Todas las problemáticas planteadas en las clases de mi carrera se resuelven con acercamientos técnicos } \\
\text { y no con teoría. }\end{array}$ \\
\hline $\mathrm{R} 6$ & $\begin{array}{l}\text { De mis conocimientos e ideas de diferentes áreas de estudio, puedo descubrir cuál es el apropiado para } \\
\text { resolver un problema específico. }\end{array}$ \\
\hline R7 & $\begin{array}{l}\text { Cuando me cuesta trabajo desarrollar una actividad en clase o tarea, desconozco cuando mi dificultad se } \\
\text { debe a que yo sólo sé pensar de la manera "en la que me enseñan a pensar en mi carrera". }\end{array}$ \\
\hline R8 & $\begin{array}{l}\text { Pocas veces reflexiono en cómo mis compañeros de clase pensaron y resolvieron algún problema para } \\
\text { mejorar mis propias soluciones. }\end{array}$ \\
\hline R9 & Veo conexiones entre mi carrera y otras áreas distintas a la mía. \\
\hline R10 & Si me pidieran, podría identificar conocimientos e ideas distintivas de otros campos de estudio. \\
\hline $\mathrm{R} 11$ & $\begin{array}{l}\text { Es muy difícil usar en otros escenarios (otras clases, mi vida cotidiana, etc.) lo que aprendo en una materia } \\
\text { en específico. }\end{array}$ \\
\hline R12 & $\begin{array}{l}\text { Puedo identificar hechos, pruebas y fundamentos en los que diferentes disciplinas académicas se } \\
\text { sustentan. }\end{array}$ \\
\hline $\mathrm{R} 13$ & $\begin{array}{l}\text { Puedo recoger ideas de otras áreas para ayudarme a entender mejor el contenido de las clases de mi } \\
\text { carrera. }\end{array}$ \\
\hline R14 & Frecuentemente me detengo a reflexionar sobre lo que estoy pensando para determinar si omito algo. \\
\hline $\mathrm{R} 15$ & Valoro leer sobre temas diferentes a los de mi carrera. \\
\hline R16 & $\begin{array}{l}\text { Usualmente estoy satisfecho(a) con la manera en la que pienso y actúo, sin detenerme a pensar en cómo } \\
\text { mejorar. }\end{array}$ \\
\hline
\end{tabular}

Tabla 2: Especificaciones del instrumento de medición, clasificados por su dimensión. (-) significa reactivo invertido

\begin{tabular}{|l|l|l|}
\hline Dimensión & Descripción & Reactivos \\
\hline $\begin{array}{l}\text { Percepción de conocimientos } \\
\text { disciplinares }\end{array}$ & $\begin{array}{l}\text { Medición de la percepción del conocimiento de límites y } \\
\text { marcos de referencia disciplinarios para la solución de } \\
\text { problemas }\end{array}$ & $2,7(-), 10,12$ \\
\hline $\begin{array}{l}\text { Percepción de habilidades } \\
\text { para la interdisciplinariedad }\end{array}$ & $\begin{array}{l}\text { Medición de la percepción de capacidades para la } \\
\text { integración natural del conocimiento }\end{array}$ & $\begin{array}{l}1,3,5(-), 6,9, \\
11(-), 13,15\end{array}$ \\
\hline $\begin{array}{l}\text { Percepción de actitudes } \\
\text { reflexivas }\end{array}$ & $\begin{array}{l}\text { Medición de la percepción de la capacidad para } \\
\text { reflexionar }\end{array}$ & $4,8(-), 14,16(-)$ \\
\hline
\end{tabular}

Se implementó el Test de percepción sobre la interdisciplinariedad a 279 estudiantes de una universidad privada del noreste de México durante octubre de $2017,76 \%$ de manera presencial y $24 \%$ en línea. De los participantes, 123 fueron del género femenino y 156 del género masculino, correspondientes a 44 y 56 por ciento respectivamente. La mayoría de los encuestados se encontraban cursando el segundo año de diversos programas académicos de nivel superior de áreas de ingeniería, negocios y ciencias de la salud. El rango de edades comprendió de los 18 a los 23 años con una moda de 19 años.

\section{RESULTADOS Y DISCUSIÓN}

De la recolección de los datos derivados de la implementación del test a 279 estudiantes, primero se realizó un análisis estadístico descriptivo y luego se revisó la validación del instrumento como confiable y válido para su uso (figura 2). El proceso de validación consistió en la fundamentación de la confiabilidad del test en su totalidad y relativa a cada dimensión, además del procedimiento de validez estructural de cada reactivo en relación a su correspondiente clasificación. Anterior al análisis descriptivo y a la validación, los datos fueron preparados transformando las puntuaciones de los reactivos invertidos. 


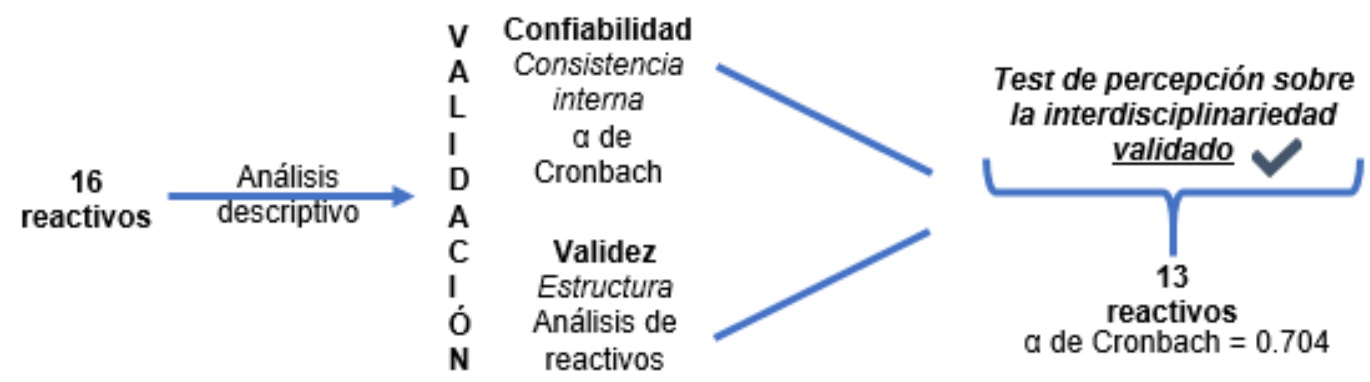

Fig. 2: Ruta de validación del Test de percepción sobre la interdisciplinariedad

\section{Análisis descriptivo}

Este análisis se enfoca a los estadísticos que describen las medidas de tendencia central y de variabilidad de las respuestas (tabla 3). Se observa que las medias de todos los reactivos excepto R16 son iguales o mayores a 3. Es decir, los encuestados se muestran favorables al entendimiento de la existencia de distintas disciplinas y a la identificación de sus respectivos conocimientos particulares, traduciéndose en percepciones positivas hacia algunas de las habilidades necesarias para la integración de conocimientos. No obstante, al observar las medianas de cada reactivo, medida de tendencia central recomendada para las escalas ordinales como la escala Likert, se observa que los reactivos $3,5,7,8$ y 16 obtuvieron respuestas centradas en una percepción neutra, ni positiva ni negativa, de acuerdo a la escala.

Tabla 3: Estadística descriptiva de la validación del Test de percepción sobre la interdisciplinariedad $(\mathrm{n}=279)$

\begin{tabular}{|c|c|c|c|c|c|}
\hline Reactivo & Media & Mediana & Varianza & Desviación estándar & Coeficiente de variación \\
\hline \multicolumn{6}{|c|}{ Dimensión 1 Conocimientos disciplinares } \\
\hline R10 & 3.900 & 4 & 0.769 & 0.847 & 0.217 \\
\hline R12 & 3.799 & 4 & 0.672 & 0.820 & 0.216 \\
\hline $\mathrm{R} 2$ & 3.656 & 4 & 0.968 & 0.984 & 0.269 \\
\hline R7 & 3.269 & 3 & 1.348 & 1.161 & 0.355 \\
\hline \multicolumn{6}{|c|}{ Dimensión 2 Habilidades interdisciplinares } \\
\hline R9 & 4.240 & 4 & 0.831 & 0.911 & 0.215 \\
\hline $\mathrm{R} 1$ & 4.151 & 4 & 0.769 & 0.877 & 0.211 \\
\hline R6 & 3.953 & 4 & 0.534 & 0.731 & 0.185 \\
\hline R13 & 3.975 & 4 & 0.838 & 0.915 & 0.230 \\
\hline R15 & 4.014 & 4 & 1.057 & 1.028 & 0.256 \\
\hline R11 & 3.606 & 4 & 1.470 & 1.212 & 0.336 \\
\hline R3 & 3.186 & 3 & 1.397 & 1.182 & 0.371 \\
\hline R5 & 3.115 & 3 & 1.411 & 1.188 & 0.381 \\
\hline \multicolumn{6}{|c|}{ Dimensión 3 Actitudes reflexivas } \\
\hline R4 & 4.240 & 4 & 0.809 & 0.900 & 0.212 \\
\hline R14 & 3.982 & 4 & 0.931 & 0.965 & 0.242 \\
\hline $\mathrm{R} 8$ & 3.065 & 3 & 1.456 & 1.207 & 0.394 \\
\hline R16 & 2.767 & 3 & 1.338 & 1.157 & 0.418 \\
\hline
\end{tabular}

El coeficiente de variación permite la comparación de la variabilidad relativa y homogeneidad de respuestas de cada reactivo, a comparación de la varianza y la desviación estándar (Lewis-Beck et al., 2004). Los reactivos $3,5,7,8,11$ y 16 resultaron ser los reactivos con un coeficiente de variación superior al resto de los ítems. 
Complementando la tabla 3 , se presenta un gráfico de frecuencias por reactivo (figura 3), ilustrativo de los resultados de la implementación. Se observa que R3, R5, R8 y R16 muestran un comportamiento proporcional de percepciones centrado en la neutralidad, además de alcanzar altos niveles de variabilidad en las percepciones que miden. Interpretando desde el significado de estos reactivos, para el caso de R3 y R5 los resultados indican heterogeneidad sobre la importancia de buscar puntos de vista distintos y ayuda específica de expertos en áreas disciplinares diferentes a su propia área de estudio, así como de percibir si su carrera les enseña a tener un pensamiento más teórico o práctico. En cuanto a R16, la interpretación del enunciado señala subjetividad respecto la comodidad de continuar acciones y evitar la reflexión. Finalmente, sobre R8, se interpretan dos escenarios opuestos (comportamiento bimodal): estudiantes que sí suelen prestar atención y reflexionar sobre las respuestas y pensamientos de sus compañeros(as) de clase con la finalidad de compararlas con sus propias respuestas y mejorar, y los que no.

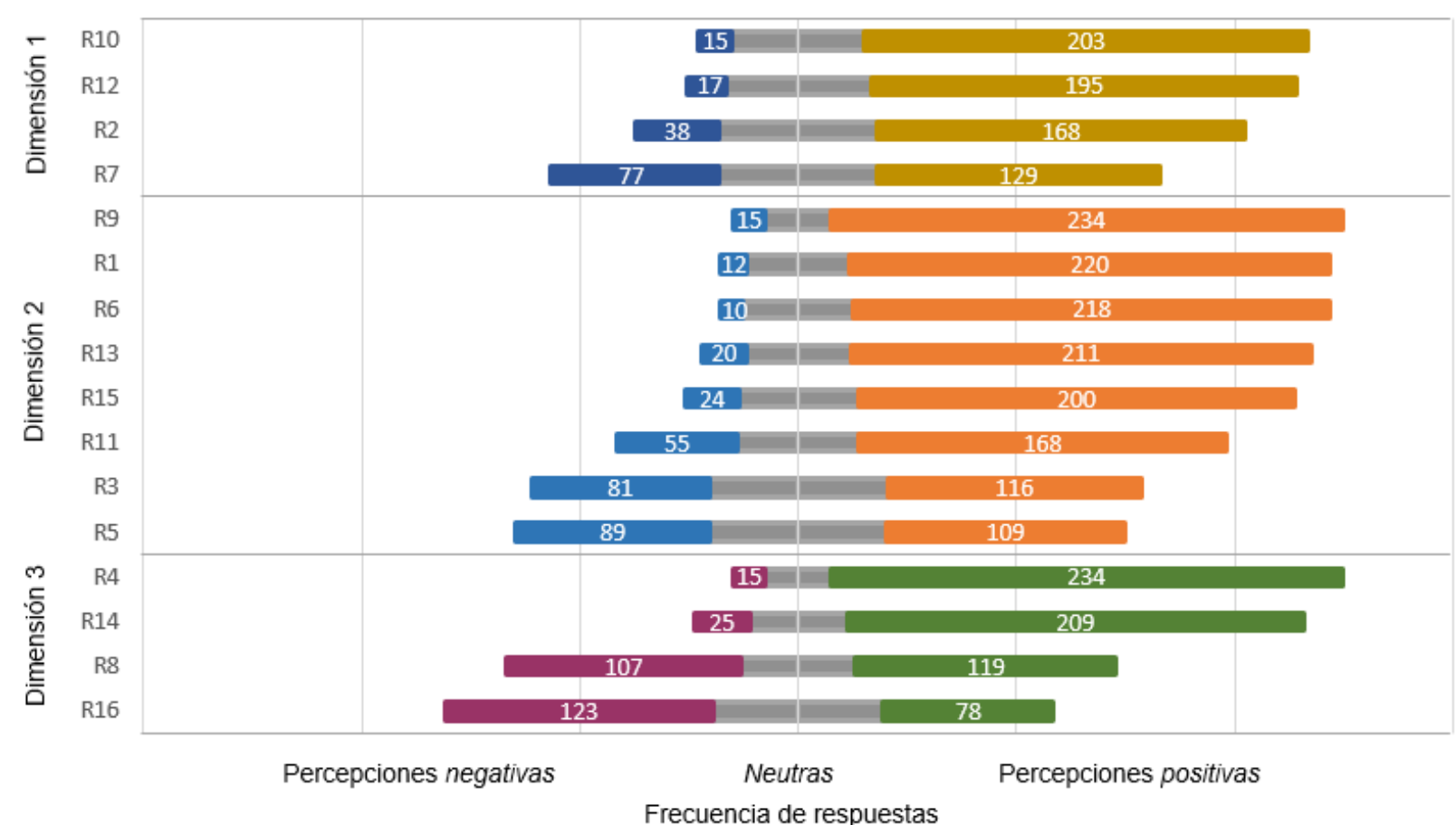

Fig. 3: Frecuencia de respuestas para cada reactivo donde las Percepciones negativas corresponden a puntuaciones en escala de Likert: 1 - Completamente en desacuerdo y 2 - En desacuerdo; Neutras a 3 - Ni de acuerdo ni en desacuerdo; y Percepciones positivas a 4- De acuerdo y 5-Completamente de acuerdo

De la figura 3 y de la tabla 3, se señalan los ítems 3, 5, 7, 8 y 16 debido a la neutralidad de las medianas, la proporción de percepciones positivas, neutras y negativas; $y$ al alto coeficiente de variación. Estos indicadores podrían señalar a los reactivos mencionados como débiles para los fines del instrumento de medición. No obstante, para la determinación de la validez y confiabilidad del instrumento existen otros métodos estadísticos para la toma de decisiones.

\section{Validación del instrumento}

La ruta de validación del instrumento (figura 2) consistió en la determinación de elementos estadísticos sobre la confiabilidad y la validez. Para la cuantificación del nivel de confiabilidad de todo el instrumento, qué tan bien mide el instrumento aquello que se pretende, y la determinación de la consistencia interna entre reactivos, se calculó el coeficiente de alfa de Cronbach. Este coeficiente puede tomar valores entre 0 y 1 , considerando que generalmente aquellos coeficientes superiores a 0.700 son suficientemente confiables para fines de investigación (Nunnally, 1967). En este caso, el valor obtenido fue de 0.660 para los 16 reactivos propuestos. Esto se interpretó como un indicador de que el instrumento debía de ser mejorado para aumentar este coeficiente por medio de la identificación de los reactivos que restan consistencia interna.

Con la finalidad de fundamentar la validez del instrumento, determinación de que el instrumento mide lo que pretende medir, se realizó un análisis de correlación con índice de homogeneidad. Con una correlación de Pearson (tabla 4), se calculó este índice entre cada ítem con respecto a la puntuación total del instrumento. Se encontró que los reactivos 3, 5 y 16 tenían un índice bajo de homogeneidad. Para fundamentar la validez estructural de todo el instrumento se llevó a cabo un análisis de reactivos, empleando el método de coeficientes de correlación entre cada reactivo y la sumatoria de puntuación en cada una de sus dimensiones comparada con la suma total del test. En este análisis señala una tendencia de pertenencia de cada ítem a 
su respectiva dimensión. Lo anterior muestra desde esta primera validación que las tres dimensiones del diseño del instrumento de Lattuca et al. (2013) no requerían de una reagrupación de reactivos, esto comprueba por medio de la implementación del nuevo instrumento propuesto que no se forzaron dimensiones por diseño, sino que se encuentra una compatibilidad natural de los constructos medidos y renombrados.

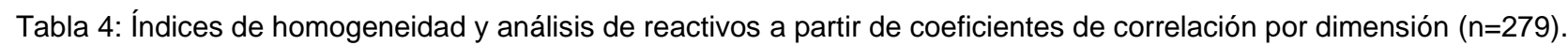
Alfa de Cronbach $=0.660$

\begin{tabular}{|c|c|c|c|c|c|c|}
\hline Dimensión & Reactivo & $\begin{array}{c}\text { Índice de } \\
\text { Homogeneidad } \\
\text { (Pearson) }\end{array}$ & $\begin{array}{c}\text { Coeficiente } \\
\text { de Spearman }\end{array}$ & $\begin{array}{l}\text { Coeficiente. } \\
\text { correlación } \\
\text { Dimensión } 1\end{array}$ & $\begin{array}{l}\text { Coeficiente } \\
\text { correlación } \\
\text { Dimensión } 2\end{array}$ & $\begin{array}{l}\text { Coeficiente. } \\
\text { correlación } \\
\text { Dimensión } 3\end{array}$ \\
\hline \multirow{4}{*}{$\begin{array}{l}\text { Dimensión } 1 \\
\text { Conocimientos } \\
\text { disciplinares }\end{array}$} & $\mathrm{R} 10$ & 0.517 & 0.450 & 0.653 & 0.403 & 0.118 \\
\hline & $\mathrm{R} 12$ & 0.539 & 0.471 & 0.643 & 0.391 & 0.207 \\
\hline & $\mathrm{R} 2$ & 0.477 & 0.374 & 0.642 & 0.387 & 0.048 \\
\hline & R7 & 0.385 & 0.220 & 0.628 & 0.167 & 0.150 \\
\hline \multirow{8}{*}{$\begin{array}{l}\text { Dimensión } 2 \\
\text { Habilidades } \\
\text { interdisciplinares }\end{array}$} & R9 & 0.514 & 0.440 & 0.356 & 0.590 & 0.121 \\
\hline & $\mathrm{R} 1$ & 0.525 & 0.410 & 0.384 & 0.573 & 0.149 \\
\hline & R6 & 0.432 & 0.424 & 0.401 & 0.422 & 0.114 \\
\hline & R13 & 0.487 & 0.404 & 0.360 & 0.550 & 0.106 \\
\hline & $\mathrm{R} 15$ & 0.422 & 0.279 & 0.226 & 0.548 & 0.068 \\
\hline & R11 & 0.412 & 0.222 & 0.213 & 0.443 & 0.211 \\
\hline & R3 & 0.310 & 0.144 & 0.041 & 0.451 & 0.100 \\
\hline & R5 & 0.223 & -0.011 & 0.068 & 0.229 & 0.178 \\
\hline \multirow{4}{*}{$\begin{array}{l}\text { Dimensión } 3 \\
\text { Actitudes reflexivas }\end{array}$} & $\mathrm{R} 4$ & 0.375 & 0.247 & 0.128 & 0.213 & 0.544 \\
\hline & $\mathrm{R} 14$ & 0.391 & 0.266 & 0.136 & 0.207 & 0.586 \\
\hline & R8 & 0.431 & 0.238 & 0.188 & 0.197 & 0.657 \\
\hline & R16 & 0.266 & 0.043 & 0.026 & 0.075 & 0.564 \\
\hline
\end{tabular}

Debido a que se busca que los reactivos conserven una correlación lineal moderada, es decir, entre 0.3 y 0.7 de correlación de Pearson (Ratner, 2009) y además una correlación de Spearman superior a 0.2, se decide eliminar los reactivos 3, 5 y 16 (tabla 4). La decisión de eliminación también es respaldada por el alto coeficiente de variabilidad presentado en el análisis descriptivo de la implementación. Los reactivos 7, 8 y 11, a pesar de haber alertado anteriormente durante el análisis descriptivo, no son eliminados del instrumento final debido a que reportan una correlación de Pearson moderada y sus coeficientes de correlación demuestran su pertenencia a las dimensiones propuestas. La supresión de los reactivos elegidos tiene consecuencias en los índices de homogeneidad y en el análisis de reactivos del instrumento (tabla 5), además de impactar en el coeficiente de alfa de Cronbach, el cual sube a 0.704 .

Tabla 5: Índices de homogeneidad y análisis de reactivos a partir de las correlaciones por dimensión del instrumento modificado $(n=279)$. Alfa de Cronbach $=0.704$

\begin{tabular}{|c|c|c|c|c|c|c|}
\hline Dimensión & Reactivo & $\begin{array}{c}\text { Índice de } \\
\text { Homogeneidad } \\
\text { (Pearson) }\end{array}$ & $\begin{array}{c}\text { Coeficiente } \\
\text { de } \\
\text { Spearman }\end{array}$ & $\begin{array}{l}\text { Coeficiente. } \\
\text { correlación } \\
\text { Dimensión } 1\end{array}$ & $\begin{array}{l}\text { Coeficiente } \\
\text { correlación } \\
\text { Dimensión } 2\end{array}$ & $\begin{array}{l}\text { Coeficiente. } \\
\text { correlación } \\
\text { Dimensión } 3\end{array}$ \\
\hline \multirow{4}{*}{$\begin{array}{l}\text { Dimensión } 1 \\
\text { Conocimientos } \\
\text { disciplinares }\end{array}$} & R10 & 0.595 & 0.568 & 0.653 & 0.469 & 0.157 \\
\hline & $\mathrm{R} 12$ & 0.592 & 0.582 & 0.643 & 0.400 & 0.249 \\
\hline & $\mathrm{R} 2$ & 0.548 & 0.525 & 0.642 & 0.403 & 0.149 \\
\hline & $\mathrm{R} 7$ & 0.348 & 0.357 & 0.628 & 0.188 & 0.062 \\
\hline \multirow{6}{*}{$\begin{array}{l}\text { Dimensión } 2 \text { Habilidades } \\
\text { interdisciplinares }\end{array}$} & $\mathrm{R} 9$ & 0.612 & 0.590 & 0.356 & 0.699 & 0.184 \\
\hline & $\mathrm{R} 1$ & 0.578 & 0.520 & 0.384 & 0.635 & 0.168 \\
\hline & R6 & 0.565 & 0.581 & 0.401 & 0.534 & 0.215 \\
\hline & R13 & 0.566 & 0.576 & 0.360 & 0.619 & 0.157 \\
\hline & $\mathrm{R} 15$ & 0.424 & 0.394 & 0.226 & 0.534 & 0.075 \\
\hline & $\mathrm{R} 11$ & 0.382 & 0.320 & 0.213 & 0.441 & 0.155 \\
\hline \multirow{3}{*}{$\begin{array}{c}\text { Dimensión } 3 \text { Actitudes } \\
\text { reflexivas }\end{array}$} & R4 & 0.410 & 0.386 & 0.128 & 0.202 & 0.661 \\
\hline & $\mathrm{R} 14$ & 0.449 & 0.422 & 0.136 & 0.215 & 0.698 \\
\hline & R8 & 0.360 & 0.340 & 0.188 & 0.147 & 0.673 \\
\hline
\end{tabular}


Tabla 6: Reactivos finales del Test de percepción sobre la interdisciplinariedad (alfa de Cronbach=0.704)

\begin{tabular}{|c|c|}
\hline Reactivo & Enunciado \\
\hline 1 & $\begin{array}{l}\text { Disfruto pensar cómo diferentes áreas de estudio abordan los mismos problemas siguiendo caminos } \\
\text { distintos. }\end{array}$ \\
\hline 2 & $\begin{array}{l}\text { Cuando un profesor experto en su materia da una explicación a una problemática, soy bueno(a) } \\
\text { identificando si el problema podría resolverse con enfoques de otras disciplinas académicas. }\end{array}$ \\
\hline 3 & Frecuentemente me detengo a pensar si voy "bien" o "mal" en el proceso de resolver un problema. \\
\hline 4 & $\begin{array}{l}\text { De mis conocimientos e ideas de diferentes áreas de estudio, puedo descubrir cuál es el apropiado para } \\
\text { resolver un problema específico. }\end{array}$ \\
\hline 5 & $\begin{array}{l}\text { Cuando me cuesta trabajo desarrollar una actividad en clase o tarea, desconozco cuando mi dificultad se } \\
\text { debe a que yo sólo sé pensar de la manera "en la que me enseñan a pensar en mi carrera". }\end{array}$ \\
\hline 6 & $\begin{array}{l}\text { Pocas veces reflexiono en cómo mis compañeros de clase pensaron y resolvieron algún problema para } \\
\text { mejorar mis propias soluciones. }\end{array}$ \\
\hline 7 & Veo conexiones entre mi carrera y otras áreas distintas a la mía. \\
\hline 8 & Si me pidieran, podría identificar conocimientos e ideas distintivas de otros campos de estudio. \\
\hline 9 & $\begin{array}{l}\text { Es muy difícil usar en otros escenarios (otras clases, mi vida cotidiana, etc.) lo que aprendo en una materia } \\
\text { en específico. }\end{array}$ \\
\hline 10 & $\begin{array}{l}\text { Puedo identificar hechos, pruebas y fundamentos en los que diferentes disciplinas académicas se } \\
\text { sustentan. }\end{array}$ \\
\hline 11 & $\begin{array}{l}\text { Puedo recoger ideas de otras áreas para ayudarme a entender mejor el contenido de las clases de mi } \\
\text { carrera. }\end{array}$ \\
\hline 12 & Frecuentemente me detengo a reflexionar sobre lo que estoy pensando para determinar si omito algo. \\
\hline 13 & Valoro leer sobre temas diferentes a los de mi carrera. \\
\hline
\end{tabular}

Contrastando la correlación antes y después de eliminar los reactivos se puede observar que los ítems continúan en la dimensión en la que están asignados pues no presentan una correlación mayor con alguna otra de las dimensiones que componen el instrumento (tabla 5). Al analizar el coeficiente de alfa de Cronbach para cada dimensión posterior a la eliminación de reactivos $(3,5$ y 16) se respalda la pertenencia de los enunciados a su dimensión correspondiente. Para la dimensión 1 el coeficiente se mantiene en 0.51 pues no se ha eliminado ningún reactivo. Para la dimensión 2 el coeficiente original es de 0.47 y después de la eliminación de los reactivos 3 y 5 , se convierte en 0.57 . Finalmente, para la dimensión 3 el coeficiente es de 0.37 y sin el reactivo 16 es de 0.39 . A partir de este segundo análisis de confiabilidad y validez del instrumento, se determina con evidencias el uso validado de trece reactivos (tabla 6 ).

Respecto a las limitaciones de la validación de este instrumento, hay que tener en cuenta que al ser un cuestionario autogestionado podría cuestionarse la subjetividad e interpretación que cada participante reporta de sí mismo; sin embargo, al tratarse de una escala de valoración a través de la identificación o no identificación, no hay respuestas que puedan considerarse como correctas o incorrectas. Si los enunciados son comprendidos por los encuestados sin prestarse a malinterpretación y ambigüedad, las preguntas son sobre eventos recientes o cotidianos, los respondientes se sienten en completa libertad de responder con sinceridad, los resultados del auto-reporte serán válidos y confiables, además de útiles para comparar percepciones entre grupos de una misma población (Volkwein et al., 2004). En cuanto la redacción de los reactivos, se cuidó la presencia de enunciados escritos tanto positiva como negativamente. Cabe señalar que dos de los tres reactivos que decidieron eliminarse por los índices de homogeneidad y su influencia en el coeficiente de alfa de Cronbach (R5 y R16 del primer test propuesto) estaban redactados de manera invertida. La debilidad adquirida por la exclusión de reactivos recae en la persistente desigualdad de ítems por dimensión, esto representa un área de oportunidad para la continuidad futura de este trabajo en busca de un instrumento para la percepción sobre la interdisciplinariedad más robusto y preciso.

\section{CONCLUSIONES}

De los resultados mostrados de la aplicación a 279 estudiantes universitarios del presente instrumento para evaluar la percepción sobre la interdisciplinariedad en la vida académica y los conocimientos, habilidades y actitudes para experimentarla, de su análisis y de su discusión, se concluye que:

1. El presente instrumento compuesto por tres dimensiones se valida su confiabilidad con un coeficiente de alfa de Cronbach de 0.704 , respaldando su consistencia interna. Este instrumento complementa los reactivos propuestos por Lattuca et al. (2013) agregando enunciados a una de las dimensiones, relativa a las actitudes para la interdisciplinariedad, del trabajo de Tutticci et al. (2017) sobre pensamiento reflexivo. 
2. Se respalda la validez estructural por medio del análisis de índice de homogeneidad de los ítems propuestos en el instrumento y del análisis de reactivos, aportando evidencias estadísticas de que el instrumento mide lo que pretende medir. Además, se verifica la pertenencia de cada ítem a su respectiva dimensión.

3. Este instrumento validado contribuye a la medición de percepciones sobre la interdisciplinariedad de estudiantes universitarios de cualquier área del conocimiento. La información que con este instrumento se recopile e interprete podría ser de utilidad para directivos de instituciones educativas, aportando argumentos que soportan la relevancia del enfoque interdisciplinario en la educación universitaria. Esto último, no sólo a partir del estudio del impacto de estos enfoques educativos como se aborda en literatura, sino desde la perspectiva del estudiante y su percepción sobre estas interacciones disciplinares, abriendo la discusión de la migración de un modelo fragmentado y atomizado en disciplinas hacia uno integrador de distintas áreas del conocimiento.

\section{AGRADECIMIENTOS}

Se extiende un agradecimiento al Dr. Jaime Ricardo Valenzuela por su asesoría en la elaboración del instrumento y a la Dra. Claudia Navarro por su colaboración en la revisión del manuscrito.

\section{REFERENCIAS}

Cezarino, L., L.B. Liboni, M.F. Oliveira y A.C.F. Caldana, Soft Systems Methodology and Interdisciplinarity in Management Education, doi: 10.1002/sres.2383, Systems Research and Behavioral Science, 33(2), 278-288 (2015)

Contreras-Velásquez, J.C., S.Y. Wilches-Duran, M.E. Graterol-Rivas y M.J. Bautista-Sandoval, Educación Superior y la Formación en Emprendimiento Interdisciplinario: Un Caso de Estudio, doi:10.4067/S0718-50062017000300003, Formación Universitaria, 10(3), 11-20 (2017)

Costa, L.S.O., V.F.A. Barros, M.C.R. Lopes y L.P. Marques, La Formación Docente y la Educación de Jóvenes y Adultos: Análisis de la Práctica Pedagógica para la Enseñanza de Ciencias, doi: 10.4067/S0718-50062015000100002, Formación Universitaria, 8(1), 3-12 (2015)

Davies, M. y M. Devlin, Chapter I, Interdisciplinary higher education; in Interdisciplinary Higher Education: Perspectives and Practicalities, vol. 5 by M. Davies, M. Devlin y M. Tight, 3-28, Emerald Group Publishing Limited, Bingley, UK (2010)

Deneme, S. y A. Selen, On Applying the Interdisciplinary Approach in Primary Schools, doi: 10.1016/j.sbspro.2012.05.217, Procedia-Social and Behavioral Sciences, 46, 885-889 (2012)

Feng, L., Teacher and Student Responses to Interdisciplinary Aspects of Sustainability Education: What Do We Really Know? doi: 10.1080/13504622.2011.574209, Environmental Education Research, 18(1), $31-43$ (2012)

Fernandes, M.T., Value Construct towards Innovation, doi: 10.7763/IJIMT.2012.V3.189, International Journal of Innovation, Management and Technology, 3(1), 10-19 (2012)

Knight, D.B., L.R. Lattuca, E.W. Kimball y R.D. Reason, Understanding Interdisciplinarity: Curricular and Organizational Features of Undergraduate Interdisciplinary Programs, doi: 10.1007/s10755-012-9232-1, Innovative Higher Education, 38(2), 143-158 (2012)

Koch, F.D., A. Dirshch-Weigand y otros tres autores, Motivating First-year University Students by Interdisciplinary Study Projects, doi: 10.1080/03043797.2016.1193126, European Journal of Engineering Education, 42(1), 17-31 (2017)

Lattuca, L., L. Voigt y K. Fath, Does Interdisciplinarity Promote Learning? Theoretical Support and Researchable Questions, doi: 10.1353/rhe.2004.0028, The Review of Higher Education, 28(1), 23-48 (2004)

Lattuca, L.R, D. Knight e I. Bergom, Developing a Measure of Interdisciplinary Competence, International Journal of Engineering Education, ISSN: 0949149X, 29(3), 726-739 (2013)

Lattuca, L.R, D. Knight y otros tres autores, Examining the Impact of Interdisciplinary Programs on Student Learning, doi: 10.1007/s10755-017-9393-z, Innovative Higher Education, 42(4), 1-17 (2017)

Ledoux, M. y N. McHenry, A constructivist approach in the interdisciplinary instruction of science and language arts methods, doi: 10.1080/1047621042000304510, Teaching Education, 15(4), 385-399 (2004)

Lenoir, Y. y A. Hasni, Interdisciplinarity in Primary and Secondary School: Issues and Perspectives, doi: 10.4236/ce.2016.716233, Creative Education, 7(16), 2433-2458 (2016)

Lewis-Beck, M.S., A. Bryman y T. Futing Liao, The SAGE encyclopedia of social science research methods, $1^{\text {a }}$ Ed., doi: 10.4135/9781412950589, Sage Publications, Inc. Thousand Oaks, California, USA (2004)

Liu, S.Y., C.S. Lin y C.C. Tsai, College Students' Scientific Epistemological Views and Thinking Patterns in socioscientific Decision Making, doi: 10.1002/sce.20422, Science Education, 95(3), 497-517 (2011)

Miles, M. y S. Rainbird. Evaluating Interdisciplinary Collaborative Learning and Assessment in the Creative Arts and Humanities, doi: 10.1177/1474022214561759, Arts and Humanities in Higher Education, 14(4), $409-425$ (2015)

Morin, E., La mente bien ordenada: repensar la reforma, reformar el pensamiento, $1^{a} \stackrel{ }{ }$ Ed., Seix Barral, Barcelona, España (2000) 
Morin, E., Los siete saberes necesarios para la educación del futuro, 1aㅡ., UNESCO, París, Francia (1999)

Nunnally, J. C., e I.H. Bernstein, Psychometric theory, Vol. 226, 3ª Ed., McGraw-Hill, New York, USA (1967)

Parker, J., Competencies for interdisciplinarity in higher education, doi: 10.1108/14676371011077559, International Journal of Sustainability in Higher Education, 11(4), 325-338 (2010)

Piaget, J., L'interisciplinarité: Problèmes D'enseignement et de Recherche dans les Universités [La Interdisciplinariedad: Problemas de Enseñanza y de Investigación dentro de las Universidades], ${ }^{a}$ Ed., OCDE, París, Francia (1972)

Ratner, B., The Correlation Coefficient: Its Values Range Between + 1/- 1, or Do They? doi: 10.1057/jt.2009.5, Journal of Targeting, Measurement and Analysis for Marketing, 17(2), 139-142 (2009)

Self, J.A. y J.J. Baek, Interdisciplinarity in Design Education: Understanding the Undergraduate Student Experience, doi: 10.1007/s10798-016-9355-2, International Journal of Technology and Design Education, 27(3), 459-480 (2017)

Tarrant, S.P. y L.P. Thiele, Enhancing and Promoting Interdisciplinarity in Higher Education, doi: 10.1007/s13412-0160402-9, Journal of Environmental Studies and Sciences, 7(2), 355-360 (2017)

Tutticci, N., P.A. Lewis y F. Coyer. Measuring third year undergraduate nursing students' reflective thinking skills and critical reflection self-efficacy following high fidelity simulation: A pilot study, doi: 10.1016/j.nepr.2016.03.001, Nurse Education in practice, 18, 52-59 (2016)

Visholm, A., L. Grosen, M. Norn y R. Jensen, Interdisciplinary Research is Key to Solving Society's Problems, DEA, Dinamarca (2012)

Volkwein, J.F., L.R. Lattuca y otros tres autores, Engineering Change: A Study of the Impact of EC2000, International Journal of Engineering Education, ISSN: 0949149X, 20(3), 318-328 (2004)

Vygotsky, L.S., Mind in society: the development of higher psychological processes, $1^{\text {a }}$ Ed., Harvard University Press, Cambridge, Massachusetts, USA (1978)

Vygotsky, L.S., Thought and Language, Rev. Edition, Massachusetts Institute of Technology Press, Cambridge, Massachusetts, USA (1962)

Walczak, M.M. y D. Van Wylen, Are "New Building" Learning Gains Sustainable? Revisiting Our Goals after Five Years, Journal of College Science Teaching, ISSN: 0047-231X, 44(6), 17-23 (2015)

Wood, K. E., Interdisciplinary Instruction for all Learners K-8: A Practical Guide, 4ª Ed., Pearson, New York, USA (2010) 
